\title{
Location-dependent User Selection Based on Sum Rate Approximation in Large System Regime for Massive MIMO
}

\author{
Vera Noviana Sulistyawan ${ }^{1}$, Rina Pudji Astuti ${ }^{1,1}$,and Arfianto Fahmi ${ }^{1}$ \\ ${ }^{1}$ Electrical Engineering, the School of Engineering, Telkom University. Bandung, Jawa \\ Barat 40257, Indonesia.
}

\begin{abstract}
Massive MIMO with multiple BS antennas can give simultaneous service for multiple user equipments (UEs) that are widely considered in massive connectivity to meet high data rate requirements. User selection is critical to optimize the overall performance of MIMO systems in various scenarios and has been extensively studied in cellular networks to guarantee service for users. In the previous study, location-dependent user selection (LUS) had extremely low computational complexity which is capable to enhance sum rate performance, but there are many environmental condition assumptions that make this algorithm does not reflect real conditions. In this research, we proposed modified LUS with approximations of sum rate in large system regimes by adding the sum ergodic of the distance from one user to another which enhance sum rate performance. In addition, we vary the user environment that was ignored in previous research by varying the path loss exponent values. In this research, we focus modify on sub-urban areas with each UEs having different environmental conditions. The selection scheme is equipped with spatial correlation fading on the transmitter side MIMO antenna. The simulation shows an increase in sum rate between 0.0012 to 0.3935 in perfect CSI. For the imperfect CSI with antenna correlation coefficient for power at $30 \mathrm{dBm}$ is 0.5 when $32 \times 64$ antennas is 14 optimal active UEs with sum rate is $23.4207 \mathrm{bps} / \mathrm{Hz}$. For cases where the user is located in different positions with different environmental circumstances, with $32 \times 64$ antennas showing the highest sum rate is 24.8436 bps/Hz with 17 optimal UEs.
\end{abstract}

\section{Introduction}

Massive device connectivity is a key requirement for next cellular network technology with many devices but few devices are active at the same time [1]. It is important to know how many optimal user equipments (UEs) that can be served at the same time with few factors to consider. The optimal number of UEs is required to guarantee the rate requirement. To fulfil the requirements of high data rate, massive MIMO with multi antenna BS can simultaneously serve multiple UEs considered to serve a large number of users simultaneously at higher rates[2]. Massive MIMO can achieve very high performance results through signal transmission with a large number of antennas on the transmitter and receiver

sides[3]. It is very important to select the active users to be served simultaneously and determine the optimal number of users to be served to achieve a high rate which is called

${ }^{1}$ Corresponding author: rinapudjiastuti@telkomuniversity.ac.id 
user selection. User selection has been extensively studied in cellular networks to guarantee user service.

In a previous research, a massive MIMO user selection was performed using an important instantaneous Channel State Information (CSI) to optimize the overall performance of MIMO massive systems in various scenarios, Semiorthogonal User Selection (SUS) using the channel orthogonality rates among UEs [4][5][6] with instantaneous CSI to improve the sum rates system, but has a high complexity when applied to massive MIMO. While to consider fairness among UEs, round-robin scheduling[4] and RUS (Random User Selection)[7] can be considered. Since they are two simple methods that offer equal opportunities to all candidate UEs. In the study[7], the proposed RUS scheme is unlike conventional Semi-orthogonal User Selection (SUS) that requires instantaneous CSI from small scale fading, but requires only long term CSI and focuses on how many UEs should be selected for simultaneous transmission so have low complexity. Location-dependent User Selection (LUS) [7] had extremely low computational complexity with approximations of sum rate in large system regimes, which are capable of significantly enhances the system sum rate performance. In the previous study, LUS had extremely low computational complexity which capable enhanced sum rate performance, but there are many environmental condition assumptions that make this algorithm does not reflect real conditions.

In this paper, we proposed modified LUS by adding the sum ergodic of the distance from one user to another using approximations of sum rate in large system regimes, which are capable of significantly enhancing the sum rate performance. In addition, we modify the condition of the user's environment which being ignored in previous research. In this paper, we focus modifying users area only for sub-urban areas with each UEs having different environmental conditions. The selection scheme is equipped with spatial correlation fading on the transmitter side MIMO antenna so as to accurately reflect the relevant phenomenon of propagation in real life. Furthermore, this paper is divided into few sections. Design of the algorithm is presented in Section 2. In Section 3, we present results and analysis of the simulation results. Finally, conclusion are drawn in Section 4.

\section{Algorithm Design}

In this paper, we present the modified LUS where here we add sum ergodic distance which increase performance. User selection is designed with sum rate approximation in a large regime system on the transmitter side. Simulation process focus on downlink transmission in single common circular cell model. From the UEs candidate side, we place the UEs and calculate the distance with the hypergeometric function. We only focus on suburban areas. We analyze the results where UEs are placed in a fixed place with the same environmental conditions and when UEs are placed in locations with different environmental circumstances. Here we vary the value of path loss exponent. This scheme can be seen in the Figure 1.

\subsection{Sum Rate Approximation in Large-System Regime}

With relying on the random matrix theory based on large system analysis can derive a deterministic approximation of the ergodic sum rate. The deterministic SINR approximations 


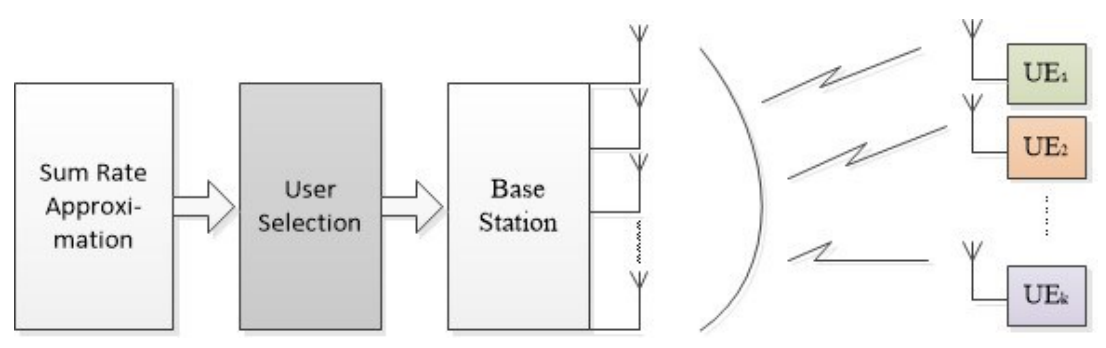

Fig 1. Location-dependent User Selection Based on Sum Rate Approximation in Large System Regime for Massive MIMO

enable us to solve various practical optimization problems[10]. The approximation of the ergodic sum rate is derived. The $\left(\operatorname{SINR}_{k}\right)$ of each user from the receiver side is defined as follows[7]:

$$
\operatorname{SINR}_{k} \stackrel{\text { a.s. }}{\rightarrow} \frac{1}{\sum_{k=1}^{K} d_{i}^{\alpha}\left(A(K, M)+B(K, M) d_{i}^{-\alpha}\right)}
$$

Each users gives feedback on the best SINR value. Where $d_{i}$ is the distance between the BS and the active user of $K$. While the $A(K, M)$ and $B(K, M)$ can be calculated by[7]:

$$
\begin{aligned}
& A(K, M)=\frac{1}{1-\rho} \frac{\sigma_{n}^{2}}{P c \phi M} \\
& B(K, M)=\frac{\rho}{1-\rho} \frac{\psi}{M \phi^{2}-K \psi}
\end{aligned}
$$

In Equation 2 and $3, \sigma^{2}$ is the total noise power and $c$ is the path loss in the reference distance of magnitude corresponding to the existing parameter in Table 1. $\rho$ is a channel estimation error where $0 \leq \rho \leq 1 . P$ is the power transmit. $M$ is the number of BS antennas and $K$ is active UEs where $(K<M)$.

From Equation 2 and Equation 3, $\phi$ and $\psi$ are the unique solution of the equation that proposed in [7]:

$$
\begin{aligned}
& \phi=\frac{1}{M} \operatorname{tr}\left(R\left(I_{M}+\frac{K}{M} \frac{1}{\phi} R\right)^{-1}\right) \\
& \psi=\frac{1}{M} \operatorname{tr}\left(R^{2}\left(I_{M}+\frac{K}{M} \frac{1}{\phi} R\right)^{-2}\right)
\end{aligned}
$$

$I_{M}$ is the identity matrix with the $(\mathrm{N} \times \mathrm{M})$ dimension. The transmit correlation matrix at the BS is modeled by the widely used $\delta-$ Kac - Muddock - Szegö matrix R with the $(\mathrm{M} \times$ M) dimension, in which $\delta$ is the antenna correlation coefficient[9]. Note that this model can range from the totally uncorrelated scenario $(\delta=0)$ to the fully correlated scenario $(\delta=$ 1) $[9]$.

\subsection{Location-dependent User Selection (LUS)}

In this research, all UEs candidate ( $N$ single antenna) are spread in a single circular cell between inner radius $\left(R_{\min }\right)$ and outer radius $\left(R_{\max }\right)$ with Base Station $(M$ antenna) at the center of the cell. Location is denoted in polar coordinates so that the UEs coordinate are $\left(r_{k}, \theta_{k}\right)$ and BS coordinate is $(0,0)$ which can be seen in Figure 2. 


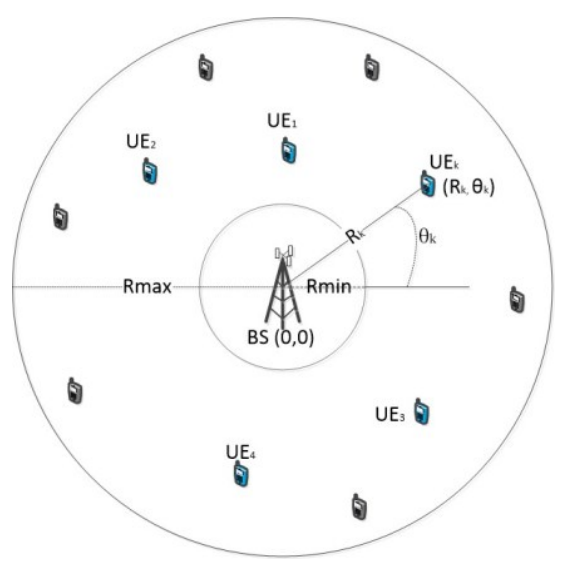

Fig 2. Location-dependent User Selection (LUS) in circular single cell with BS and candidate UEs

At LUS, we select the number of corresponding UEs in the order of large scale fading be dominated by path-loss (i.e., in ascending order of the BS-UE distance) for simultaneous data transmissions. UEs candidates located close to the BS are more likely to be active and selected than UEs located far from the BS. Therefore, with the LUS algorithm can decrease the distance. Meanwhile, due to the shortened distance between base station and terminals, transmission loss will be reduced, thus greatly improving the power efficiency[11]. Therefore $\mathfrak{R}_{L U S}$ which contains sum ergodic values of distances in $\mathrm{x}$ and y can formulated as:

$$
\Re_{L U S}=\left(1-\frac{K}{T}\right) \sum_{k=1}^{K} \int_{R_{\min }}^{R_{\max }} \log _{2}\left\{1+\frac{1-\rho}{x+y}\right\} \frac{2 r_{k}\left(R_{\max }^{2}-r_{k}^{2}\right)^{N-k}\left(r_{k}^{2}-R_{\min }^{2}\right)^{k-1}}{B(k, N-k+1)\left(R_{\max }^{2}-R_{\min }^{2}\right)^{N}} d r_{k}
$$

From Equation 6, $\rho$ is the channel estimate accuracy value whose value is already set at $0 \leq$ $\rho \leq 1$. In the case of massive MIMO where the number of Base Station (BS) antennas is greater than the number of single-antenna users, to estimate the channels, time-division duplex (TDD) operation with the reverse-link pilots are used.

$$
x=\frac{\sigma_{n}^{2}}{P c \phi M}\left\{r_{k}^{\alpha}+\sum_{i=1, i \neq k}^{K} R_{\min ^{2}}^{\alpha} F_{1}\left(k ;-\frac{\alpha}{2} ; N+1 ; 1-\frac{R_{\max }^{2}}{R_{\min }^{2}}\right)\right\}
$$

From Equation 7, there are several parameters such as $\sigma_{n}^{2}$ where is this is noise power, c is the path loss in reference distance, $\mathrm{M}$ is the number of $\mathrm{BS}$ antennas and $\mathrm{N}$ is the number of single-antenna candidate UEs.

$$
y=\frac{\rho \psi}{M \phi^{2}-K \psi}\left\{\sum_{i=1, i \neq k}^{K} r_{k}^{-\alpha} R_{\text {min }^{2}}^{\alpha} F_{1}\left(k ;-\frac{\alpha}{2} ; N+1 ; 1-\frac{R_{\max }^{2}}{R_{\min }^{2}}\right)+1\right\}
$$

The optimal amount of UE presented with the maximal sum rate can be written as:

$$
K_{L U S}^{*}=\operatorname{argmax}_{K \epsilon(1, \ldots, M-1)} \Gamma_{2}\left(T, M, K, R_{\text {min }}, R_{\text {max }}, P, c, \sigma_{n}^{2}, \rho, \alpha, \delta\right)
$$

Where $\Re_{L U S}=\Gamma_{2}\left(T, M, K, R_{\text {min }}, R_{\text {max }}, P, c, \sigma_{n}^{2}, \rho, \alpha, \delta\right)$, the value of $K_{L U S}^{*}$ is obtained when $\mathfrak{R}_{\text {LUS }}$ maximum. 


\section{Result and Analysis}

Refers to the cellular model based on [8]. The simulation will be analyzed using the following parameters in the Table 1.

Table 1. Parameters for simulation

\begin{tabular}{|c|c|}
\hline Parameters & Value \\
\hline M-antenna BS & 32 \\
\hline N single-antenna candidate UEs & 64 \\
\hline Power $(P)$ & $30 \mathrm{dBm}$ \\
\hline Number of symbols in a coherence slot $(T)$ & 196 \\
\hline Radius minimum $\left(R_{\min }\right)$ & $35 \mathrm{~m}$ \\
\hline Radius maximum $\left(R_{\max }\right)$ & $250 \mathrm{~m}$ \\
\hline Path loss in reference distance $(c)$ & $10^{-3.53}$ \\
\hline Noise power $\left(\sigma_{n}^{2}\right)$ & $-96 \mathrm{dBm}$ \\
\hline Antenna correlation coefficient $(\delta)$ & 0.5 \\
\hline Estimated channel accuracy $(\rho)$ & 0.2 \\
\hline
\end{tabular}

Using the parameter in Table 1, we obtained the value of correlation matrix on the transmitter side. In this research just considering correlation among transmit antenna BS. Correlation among receive antennas is independent of the correlation between transmit antennas. Therefore the matrix for 32x64 antennas is obtained:

$$
R_{32 \times 32}=\left[\begin{array}{cccccc}
1 & 0.5000 & 0.2500 & 0.1250 & \cdots & 4.6566 \times 10^{-10} \\
0.5000 & 1 & 0.5000 & 0.2500 & \ddots & 9.3132 \times 10^{-10} \\
0.2500 & 0.5000 & 1 & 0.5000 & \ddots & 1.8626 \times 10^{-9} \\
0.1250 & 0.2500 & 0.5000 & 1 & \ddots & \vdots \\
\vdots & \ddots & \ddots & \ddots & \ddots & 0.5000 \\
4.6566 \times 10^{-10} & \cdots & 0.1250 & 0.2500 & 0.5000 & 1
\end{array}\right]
$$

$\varphi$ and $\psi$ represent the unique solution value in the large-system regime. From simulation, we can get the value of $\varphi=0.0192$ and $\psi=3.6868 \times 10^{-4}$. If the value of the antenna correlation coefficient increased, then the value of $\varphi$ and $\psi$ will also increase but the sum rate will decrease. Therefore, when the correlation is higher then the sum rate decreases.

Simulation results can be seen from Table 2. Where LUS0 is the result of the LUS algorithm without any modification. LUS1 is the result of a modified LUS algorithm by adding sum ergodic from distance which is proven to improve performance. LUS0 and LUS1 are run under the same conditions. While LUS2 is a modification algorithm that runs on imperfect CSI condition where correlation is present in this scheme. LUS3 is the most real condition telling the state of the environment. At this time, we vary the path loss exponent value in

Table 2. Sum rate of simulation results in $\mathrm{bps} / \mathrm{Hz}$

\begin{tabular}{|c|c|c|c|c|c|c|c|c|c|}
\hline K & LUS0 & LUS1 & LUS2 & LUS3 & K & LUS0 & LUS1 & LUS2 & LUS3 \\
\hline 1 & 13.9789 & 14.3724 & 6.2382 & 6.2462 & 17 & 74.3189 & 74.3289 & 22.6756 & 24.8436 \\
\hline 2 & 24.6197 & 24.8361 & 9.7089 & 9.8131 & 18 & 73.3616 & 73.3705 & 22.1126 & 24.0298 \\
\hline 3 & 33.4901 & 33.6347 & 12.4374 & 12.4914 & 19 & 71.9682 & 71.9762 & 20.3926 & 23.0858 \\
\hline 4 & 41.0451 & 41.1493 & 14.6629 & 14.6600 & 20 & 70.1347 & 70.1417 & 20.5157 & 23.0374 \\
\hline 5 & 47.5355 & 47.6141 & 16.5184 & 16.3958 & 21 & 67.8522 & 67.8585 & 19.4911 & 21.9191 \\
\hline
\end{tabular}




\begin{tabular}{|c|c|c|c|c|c|c|c|c|c|}
\hline 6 & 53.1233 & 53.1845 & 18.0819 & 17.9909 & 22 & 65.1067 & 65.1124 & 18.3204 & 21.5407 \\
\hline 7 & 57.9223 & 57.9710 & 19.4026 & 19.5575 & 23 & 61.8781 & 61.8832 & 17.0024 & 20.0371 \\
\hline 8 & 62.0162 & 62.0558 & 20.5097 & 20.4332 & 24 & 58.1381 & 58.1427 & 15.5511 & 19.6291 \\
\hline 9 & 65.4692 & 65.5019 & 21.4241 & 21.4594 & 25 & 53.8485 & 53.8527 & 13.9663 & 18.2946 \\
\hline 10 & 68.3314 & 68.3588 & 22.1586 & 21.7955 & 26 & 48.9575 & 48.9612 & 12.2563 & 16.9970 \\
\hline 11 & 70.6426 & 70.6657 & 22.7210 & 23.2224 & 27 & 43.3947 & 43.3979 & 10.4315 & 15.3220 \\
\hline 12 & 72.4344 & 72.4542 & 23.1169 & 23.6526 & 28 & 37.0624 & 37.0653 & 8.5066 & 12.6050 \\
\hline 13 & 73.7323 & 73.7494 & 23.3496 & 23.7560 & 29 & 29.8230 & 29.8254 & 6.4877 & 10.4667 \\
\hline 14 & 74.5563 & 74.5711 & 23.4207 & 24.1961 & 30 & 21.4741 & 21.4760 & 4.3881 & 7.5631 \\
\hline 15 & 74.9218 & 74.9347 & 23.3310 & 24.0356 & 31 & 11.7044 & 11.7056 & 2.2218 & 4.0176 \\
\hline 16 & 74.8402 & 74.8515 & 23.0834 & 23.8680 & & & & & \\
\hline
\end{tabular}

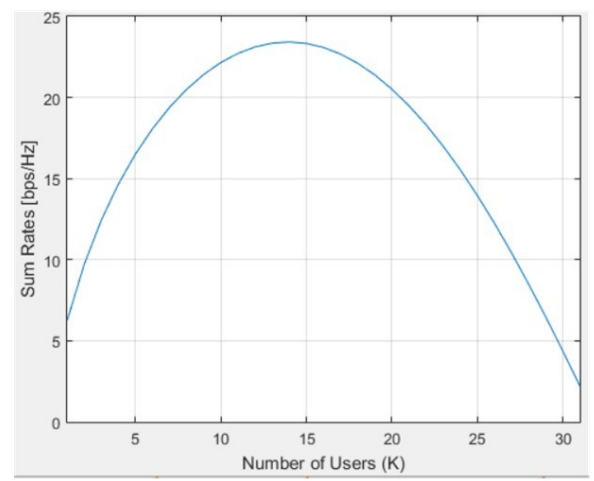

(a)

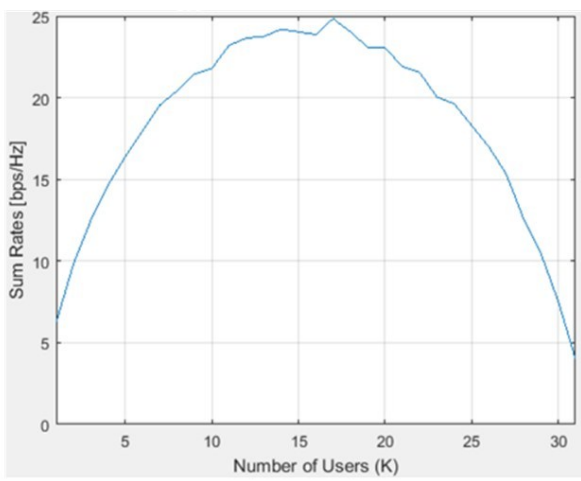

(b)

Fig 3. (a) The influence of UEs active $(K)$ to the sum rate at the fix condition (b) The influence of UEs active (K) to the sum rate at different environmental for 300 iterations

sub-urban areas where the value is 3 to 3.5. Sum rate values of LUS2 and LUS3 can be seen in Figure 3 a and b, respectively.

The graph of sum rate values can be seen in Figure 3 in point (a) and (b). In Figure 3a, the number of optimal active UEs $\left(K^{*}\right)$ which can be served to reach the optimal sum rate is 14 with sum rate is $23.4207 \mathrm{bit} / \mathrm{s} / \mathrm{Hz}$. The sum rate value decreases when correlation is present. In Figure 3a, the candidate UEs have a fix environmental condition with path loss exponent value is 3.76. The number of active UEs candidates that can be served is still similar as in previous studies when imperfect CSI can be seen in Figure 3a. However, the results of this simulation show an increase in the sum rate value than the previous one.

In Figure $3 b$, for case where the user is located in different positions with different environmental circumstances showing the highest sum rate is $24.8436 \mathrm{bps} / \mathrm{Hz}$ with 17 optimal UEs. In this situation, each UEs candidate is dispersed into various areas in the suburban area with a path loss exponent value in the range of 3 to 3.5. This scheme is very different from the scheme in previous research, where in previous studies only provided one condition in all UEs. The number of optimal UEs that can be served depends on environmental circumstances. If the environment is good, then the sum rate will be higher 
than the bad environmental conditions. The sum rate of 300 iterations is the average value of conditions repeatedly made in 300 times.

\section{Conclusion}

With the proposed LUS scheme, how many UEs to be served can be determined so that it will guarantee the rate for each UEs. The simulation results show that the optimal number of active UEs candidates is about half of the number of BS antennas. The result of our research have better performance in sum rate compared to previous research. For imperfect CSI conditions for every UEs are put in the same environmental conditions, the number of active UEs that can be served optimally is still the same as in previous research. Our research show an increase in sum rate. As for imperfect conditions and different environmental circumstances resulting in optimal number of active UEs and a sum rate corresponding to the circumstances of the environment. Better environment is needed to achieve optimal served active UEs and higher sum rate. To meet the demand for high data rates with fixed energy, the proposed scheme can be considered for massive device connectivity in $5 \mathrm{G}$ mobile communications.

\section{References}

1. H. Papadopoulos, C. Wang, O. Bursalioglu, and X. Hou, "Massive MIMO Technologies and Challenges towards 5G," no. 3, pp. 602-621, 2016.

2. N. Krishnan, R. D. Yates, and N. B. Mandayam, "Cellular Systems with Many Antennas : Large System Analysis under Pilot Contamination.”, 2013.

3. H. Papadopoulos, C. Wang, O. Bursalioglu, and X. Hou, "Massive MIMO Technologies and Challenges towards 5G," no. 3, pp. 602-621, 2016.

4. T. Yoo, S. Member, and A. Goldsmith, "On the Optimality of Multiantenna Broadcast Scheduling Using Zero-Forcing Beamforming,” vol. 24, no. 3, pp. 528-541, 2006.

5. X. Chen, Z. Zhang, and C. Yuen, "Resource Allocation for Cost Minimization in Limited Feedback MU-MIMO Systems with Delay Guarantee,” pp. 1-20, 2012.

6. K. T. K. Cheung, S. Member, S. Yang, M. IEEE, and L. Hanzo, "Spectral and Energy Spectral Efficiency Optimization of Joint Transmit and Receive Beamforming Based Multi-Relay MIMO-OFDMA Cellular Networks," pp. 1-20, 2014.

7. H. Liu, H. Gao, S. Yang, T. Lv, and S. Member, "Low-Complexity Downlink User Selection for Massive MIMO Systems,” pp. 1-12, 2015.

8. E. Björnson, L. Sanguinetti, and J. Hoydis, "Optimal Design of Energy-Efficient MultiUser MIMO Systems : Is Massive MIMO the Answer ?," vol. 14, no. 6, pp. 3059-3075, 2015.

9. Van Zelst and J. S. Hammerschmidt, "A single coefficient spatial correlation model for multiple-input multiple-output (mimo) radio channels," no. 1, pp. 2-5, 2002.

10. S. Wagner, S. Member, R. Couillet, and D. T. M. Slock, "Large System Analysis of Linear Precoding in Correlated MISO Broadcast Channels under Limited Feedback," pp. 1-28, 2012.

11. Y. Yang, J. Xu, G. Shi, and C. Wang, "5G Wireless Systems Simulation and Evaluation Techniques," 2018. 